DFTT-44/99

October 1999

\title{
Gravity on Finite Groups
}

\author{
Leonardo Castellani \\ Dipartimento di Scienze e Tecnologie Avanzate, East Piedmont University, Italy; \\ Dipartimento di Fisica Teorica and Istituto Nazionale di Fisica Nucleare \\ Via P. Giuria 1, 10125 Torino, Italy. \\ castellani@to.infn.it
}

\begin{abstract}
Gravity theories are constructed on finite groups $G$. A self-consistent review of the differential calculi on finite $G$ is given, with some new developments. The example of a bicovariant differential calculus on the nonabelian finite group $S_{3}$ is treated in detail, and used to build a gravity-like field theory on $S_{3}$.
\end{abstract}

Supported in part by EEC under TMR contract ERBFMRX-CT96-0045 


\section{Introduction}

The algebraic treatment of differential calculus in terms of Hopf structures allows to extend the usual differential geometric quantities (connection, curvature, metric, vielbein etc.) to a variety of interesting spaces that include quantum groups, noncommutative spacetimes (i.e. quantum cosets), and discrete spaces.

In this paper we concentrate on a particular sort of discrete spaces, i.e. finite group "manifolds". As we will discuss, these spaces can be visualized as collections of points, corresponding to the finite group elements, and connected by oriented links according to the particular differential calculus we build on them. Although functions $f \in \operatorname{Fun}(G)$ on finite groups $G$ commute, the calculi that are constructed on $\operatorname{Fun}(G)$ by algebraic means are in general noncommutative, in the sense that differentials do not commute with functions, and the exterior product does not coincide with the usual antisymmetrization of the tensor product.

The physical motivations for finding differential calculi on finite groups are at least threefold in our opinion:

i) the possibility of using finite group spaces as internal spaces for KaluzaKlein compactifications of supergravity or superstring theories. Harmonic analysis on such spaces is far simpler than on the usual smooth manifolds (coset spaces, Calabi-Yau spaces, etc.) or orbifolds. We note in this respect that compactification of $D=5$ Yang-Mills theory on the finite group space $Z^{2}$ yields precisely the Higgs potential, and gives it a geometric raison d' être. In fact Connes' reconstruction of the standard model in terms of noncommutative geometry [1] can be presumably recovered as Kaluza-Klein compactification of Yang-Mills theory on an appropriate discrete internal space.

ii) field theories on discrete structures are interesting per se: many statistical models are of this sort and the tools offered by differential calculi on these structures can be of use in the study of integrable models, see for ex. ref. [2].

iii) Gauge and gravity theories on finite group spaces may be used as lattice approximations. For example the action for pure Yang-Mills $\int F \wedge^{*} F$ considered on the finite group space $Z^{N} \times Z^{N} \times Z^{N} \times Z^{N}$, yields the usual Wilson action of lattice gauge theories, and $N \rightarrow \infty$ gives the continuum limit [3]. New lattice theories can be found by choosing different finite groups.

Here we propose an action for a toy theory of gravity on the smallest nonabelian finite group $S_{3}$. In fact the same type of action can be used for any finite group. Taking $Z^{N} \times Z^{N} \times Z^{N} \times Z^{N}$ yields a discretized version of gravity, in the same spirit of ref.s [4] ( where however no action principle was used).

In Section 2 a review of the differential calculus on finite groups is presented. Most of this material is not new, and draws on the treatment of ref.s [5, 6], where the 
Hopf algebraic approach of Woronowicz [7] for the construction of differential calculi is adapted to the setting of finite groups. Some developments on Lie derivative, diffeomorphisms and integration are new. The general theory is illustrated in the case of $S_{3}$ in Section 3. The "softening" of the rigid finite group manifold is discussed in Sect. 4, together with the application to a gravity-like field theory on $S_{3}$.

\section{Differential calculus on finite groups}

\section{1 $\operatorname{Fun}(\mathrm{G})$ as a Hopf algebra}

Let $G$ be a finite group of order $n$ with generic element $g$ and unit $e$. Consider Fun $(G)$, the set of complex functions on $G$. An element $f$ of $F u n(G)$ is specified by its values $f_{g} \equiv f(g)$ on the group elements $g$, and can be written as

$$
f=\sum_{g \in G} f_{g} x^{g}, \quad f_{g} \in \boldsymbol{C}
$$

where the functions $x^{g}$ are defined by

$$
x^{g}\left(g^{\prime}\right)=\delta_{g^{\prime}}^{g}
$$

Thus $\operatorname{Fun}(G)$ is a n-dimensional vector space, and the $n$ functions $x^{g}$ provide a basis. $\operatorname{Fun}(G)$ is also a commutative algebra, with the usual pointwise sum and product $[(f+h)(g)=f(g)+h(g),(f \cdot h)(g)=f(g) h(g),(\lambda f)(g)=\lambda f(g), f, h \in$ $\operatorname{Fun}(G), \lambda \in \boldsymbol{C}]$ and unit $I$ defined by $I(g)=1, \forall g \in G$. In particular:

$$
x^{g} x^{g^{\prime}}=\delta_{g, g^{\prime}} x^{g}, \quad \sum_{g \in G} x^{g}=I
$$

The $G$ group structure induces a Hopf algebra structure on $F u n(G)$, with coproduct $\Delta$, coinverse $\kappa$ and counit $\varepsilon$ defined by group multiplication, inverse and unit as:

$$
\begin{array}{ll}
\Delta(f)\left(g, g^{\prime}\right)=f\left(g g^{\prime}\right), & \Delta: \operatorname{Fun}(G) \rightarrow \operatorname{Fun}(G) \otimes \operatorname{Fun}(G) \\
\kappa(f)(g)=f\left(g^{-1}\right), & \kappa: F u n(G) \rightarrow F u n(G) \\
\varepsilon(f)=f(e), & \varepsilon: F u n(G) \rightarrow C
\end{array}
$$

In the first line we have used $F u n(G \times G) \approx F u n(G) \otimes F u n(G)$ [indeed a basis for functions on $G \times G$ is given by $\left.x^{g_{1}} \otimes x^{g_{2}}, g_{1}, g_{2} \in G\right]$. On the basis functions $x^{g}$ the costructures take the form:

$$
\Delta\left(x^{g}\right)=\sum_{h \in G} x^{h} \otimes x^{h^{-1} g}, \quad \kappa\left(x^{g}\right)=x^{g^{-1}}, \quad \varepsilon\left(x^{g}\right)=\delta_{e}^{g}
$$

The coproduct is related to the pullback induced by left or right multiplication of $G$ on itself. Consider the left multiplication by $g_{1}$ :

$$
L_{g_{1}} g_{2}=g_{1} g_{2}, \quad \forall g_{1}, g_{2} \in G
$$


This induces the left action (pullback) $\mathcal{L}_{g_{1}}$ on Fun $(G)$ :

$$
\left.\mathcal{L}_{g_{1}} f\left(g_{2}\right) \equiv f\left(g_{1} g_{2}\right)\right|_{g_{2}}, \quad \mathcal{L}_{g_{1}}: \text { Fun }(G) \rightarrow \text { Fun }(G)
$$

where $\left.f\left(g_{1} g_{2}\right)\right|_{g_{2}}$ means $f\left(g_{1} g_{2}\right)$ seen as a function of $g_{2}$. For the basis functions we find easily:

$$
\mathcal{L}_{g_{1}} x^{g}=x^{g_{1}^{-1} g}
$$

Introducing the mapping $\mathcal{L}: F u n(G) \rightarrow F u n(G \times G) \approx F u n(G) \otimes F u n(G)$ :

$$
(\mathcal{L} f)\left(g_{1}, g_{2}\right) \equiv\left(\mathcal{L}_{g_{1}} f\right)\left(g_{2}\right)=\left.f\left(g_{1} g_{2}\right)\right|_{g_{2}}
$$

we see that

$$
\mathcal{L}=\Delta
$$

Thus the coproduct mapping $\Delta$ on the function $f$ encodes the information on all the left actions $\mathcal{L}_{g}, g \in G$ applied to $f$, without reference to a particular $g$ ("point of the group manifold"). It also encodes the information on right actions.

Indeed one can define the right action $\mathcal{R}$ on $\operatorname{Fun}(G)$ as:

$$
(\mathcal{R} f)\left(g_{1}, g_{2}\right) \equiv\left(\mathcal{R}_{g_{1}} f\right)\left(g_{2}\right)=\left.f\left(g_{2} g_{1}\right)\right|_{g_{2}}
$$

Introducing the flip operator $\tau: F u n(G \times G) \rightarrow F u n(G \times G)$ :

$$
(\tau u)\left(g_{1}, g_{2}\right) \equiv u\left(g_{2}, g_{1}\right), \quad u \in F u n(G \times G)
$$

it is easy to find that:

$$
\mathcal{R}=\tau \circ \Delta
$$

For the basis functions:

$$
\mathcal{R}_{g_{1}} x^{g}=x^{g g_{1}^{-1}}, \quad \mathcal{R} x^{g}=\tau \circ \Delta\left(x^{g}\right)=\sum_{h \in G} x^{h^{-1} g} \otimes x^{h}
$$

Finally:

$$
\mathcal{L}_{g_{1}} \mathcal{L}_{g_{2}}=\mathcal{L}_{g_{1} g_{2}}, \quad \mathcal{R}_{g_{1}} \mathcal{R}_{g_{2}}=\mathcal{R}_{g_{2} g_{1}}, \quad \mathcal{L}_{g_{1}} \mathcal{R}_{g_{2}}=\mathcal{R}_{g_{2}} \mathcal{L}_{g_{1}}
$$

\section{$2.2 \quad$ First order differential calculus}

Differential calculi can be constructed on Hopf algebras $A$ by algebraic means, using the costructures of $A[7]$. In the case of finite groups $G$, differential calculi on $A=\operatorname{Fun}(G)$ have been discussed in ref.s [5, 6]. Here we review some of the results, and present new developments.

A first-order differential calculus on $A$ is defined by

i) a linear map $d: A \rightarrow \Gamma$, satisfying the Leibniz rule

$$
d(a b)=(d a) b+a(d b), \quad \forall a, b \in A ;
$$


The "space of 1-forms" $\Gamma$ is an appropriate bimodule on $A$, which essentially means that its elements can be multiplied on the left and on the right by elements of $A$ [more precisely $A$ is a left module if $\forall a, b \in A, \forall \rho, \rho^{\prime} \in \Gamma$ we have: $a\left(\rho+\rho^{\prime}\right)=$ $a \rho+a \rho^{\prime},(a+b) \rho=a \rho+b \rho, a(b \rho)=(a b) \rho, I \rho=\rho$. Similarly one defines a right module. A left and right module is a bimodule if $a(\rho b)=(a \rho) b]$. From the Leibniz rule $d a=d(I a)=(d I) a+I d a$ we deduce $d I=0$.

ii) the possibility of expressing any $\rho \in \Gamma$ as

$$
\rho=\sum_{k} a_{k} d b_{k}
$$

for some $a_{k}, b_{k}$ belonging to $A$.

To build a first order differential calculus on $\operatorname{Fun}(G)$ we need to extend the algebra $A=\operatorname{Fun}(G)$ to a differential algebra of elements $x^{g}, d x^{g}$ (it is sufficient to consider the basis elements and their differentials). Note however that the $d x^{g}$ are not linearly independent. In fact from $0=d I=d\left(\sum_{g \in G} x^{g}\right)=\sum_{g \in G} d x^{g}$ we see that only $n-1$ differentials are independent. Every element $\rho=a d b$ of $\Gamma$ can be expressed as a linear combination (with complex coefficients) of terms of the type $x^{g} d x^{g^{\prime}}$. Moreover $\rho b \in \Gamma$ (i.e. $\Gamma$ is also a right module) since the Leibniz rule and the multiplication rule (2.3) yield the commutations:

$$
d x^{g} x^{g^{\prime}}=-x^{g} d x^{g^{\prime}}+\delta_{g^{\prime}}^{g} d x^{g}
$$

allowing to reorder functions to the left of differentials. There are $n(n-1)$ independent terms $x^{g} d x^{g^{\prime}}$, since there are $n-1$ independent $d x^{g}$. A convenient independent set was chosen in ref. [5] by taking all the terms $e^{g, g^{\prime}} \equiv x^{g} d x^{g^{\prime}}$ with $g \neq g^{\prime}$. Within this set one can choose any subset, defining a consistent first order differential algebra of elements $x^{g}, e^{g, g^{\prime}}$. These different choices can be described by oriented graphs, whose vertices are elements $g$ of $G$, and where an oriented line from $g$ to $g^{\prime}$ means that the term $x^{g} d x^{g^{\prime}}$ exists in the subset [5].

\section{Partial derivatives, "curved" indices}

Consider the differential of a function $f \in F u n(g)$ :

$$
d f=\sum_{g \in G} f_{g} d x^{g}=\sum_{g \neq e} f_{g} d x^{g}+f_{e} d x^{e}=\sum_{g \neq e}\left(f_{g}-f_{e}\right) d x^{g} \equiv \sum_{g \neq e} \partial_{g} f d x^{g}
$$

We have used $d x^{e}=-\sum_{g \neq e} d x^{g}$ (from $\sum_{g \in G} d x^{g}=0$ ). The partial derivatives of $f$ have been defined in analogy with the usual differential calculus, and are given by

$$
\partial_{g} f=f_{g}-f_{e}=f(g)-f(e)
$$

Not unexpectedly, they take here the form of finite differences (discrete partial derivatives at the origin $e$ ). The partial derivatives satisfy the modified Leibniz rule:

$$
\partial_{g}\left(f f^{\prime}\right)=\left(\partial_{g} f\right) f^{\prime}(g)-f(e) \partial_{g} f^{\prime}
$$




\subsection{Left and right covariance}

A differential calculus is left or right covariant if the left or right action of $G\left(\mathcal{L}_{g}\right.$ or $\mathcal{R}_{g}$ ) commutes with the exterior derivative $d$. Requiring left and right covariance in fact defines the action of $\mathcal{L}_{g}$ and $\mathcal{R}_{g}$ on differentials: $\mathcal{L}_{g} d b \equiv d\left(\mathcal{L}_{g} b\right), \forall b \in F u n(G)$ and similarly for $\mathcal{R}_{g} d b$. More generally, on elements of $\Gamma$ (one-forms) we define $\mathcal{L}_{g}$ as:

$$
\mathcal{L}_{g}(a d b) \equiv\left(\mathcal{L}_{g} a\right) \mathcal{L}_{g} d b=\left(\mathcal{L}_{g} a\right) d\left(\mathcal{L}_{g} b\right)
$$

and similar for $\mathcal{R}_{g}$. For example the left and right action on the differentials $d x^{g}$ is given by:

$$
\mathcal{L}_{g}\left(d x^{g_{1}}\right) \equiv d\left(\mathcal{L}_{g} x^{g_{1}}\right)=d x^{g^{-1} g_{1}}, \quad \mathcal{R}_{g}\left(d x^{g_{1}}\right) \equiv d\left(\mathcal{R}_{g} x^{g_{1}}\right)=d x^{g_{1} g^{-1}}
$$

In the same spirit as in the previous section, we can introduce mappings $\Delta_{L}: \Gamma \rightarrow$ $A \otimes \Gamma$ and $\Delta_{R}: \Gamma \rightarrow \Gamma \otimes A$ that encode the information about all left or right translations:

$$
\begin{aligned}
& \Delta_{L}(a \rho b)=\Delta(a) \Delta_{L}(\rho) \Delta(b), \quad \Delta_{L}(d b)=(i d \otimes d) \Delta(b) \quad \forall a, b \in A, \rho \in \Gamma \\
& \Delta_{R}(a \rho b)=\Delta(a) \Delta_{R}(\rho) \Delta(b), \quad \Delta_{R}(d b)=(d \otimes i d) \Delta(b) \quad \forall a, b \in A, \rho \in \Gamma
\end{aligned}
$$

To see their relation with $\mathcal{L}_{g}$ and $\mathcal{R}_{g}$, consider their action on the basic terms $x^{g_{1}} d x^{g_{2}} \in \Gamma$ :

$$
\begin{gathered}
\Delta_{L}\left(x^{g_{1}} d x^{g_{2}}\right)=\Delta\left(x^{g_{1}}\right)(i d \otimes d) \Delta\left(x^{g_{2}}\right)=\sum_{h \in G} x^{h} \otimes x^{h^{-1} g_{1}} d x^{h^{-1} g_{2}} \\
\Delta_{R}\left(x^{g_{1}} d x^{g_{2}}\right)=\Delta\left(x^{g_{1}}\right)(d \otimes i d) \Delta\left(x^{g_{2}}\right)=\sum_{h \in G} x^{g_{1} h} d x^{g_{2} h} \otimes x^{h^{-1}}
\end{gathered}
$$

Defining $(f \otimes \rho)[g] \equiv f(g) \rho \equiv(\rho \otimes f)[g]$, with $f \in A=F u n(G), \rho \in \Gamma, g \in G$, we deduce:

$$
\begin{aligned}
& \Delta_{L}\left(x^{g_{1}} d x^{g_{2}}\right)[g]=x^{g^{-1} g_{1}} d x^{g^{-1} g_{2}}=\mathcal{L}_{g}\left(x^{g_{1}} d x^{g_{2}}\right) \\
& \Delta_{R}\left(x^{g_{1}} d x^{g_{2}}\right)[g]=x^{g_{1} g^{-1}} d x^{g_{2} g^{-1}}=\mathcal{R}_{g}\left(x^{g_{1}} d x^{g_{2}}\right)
\end{aligned}
$$

so that the relations we were looking for are simply

$$
\Delta_{L}(\rho)[g]=\mathcal{L}_{g} \rho, \Delta_{R}(\rho)[g]=\mathcal{R}_{g} \rho, \forall \rho \in \Gamma .
$$

Computing $\Delta_{L}$ and $\Delta_{R}$ on the basic differentials yields:

$$
\begin{aligned}
& \Delta_{L}\left(d x^{g_{1}}\right) \equiv(i d \otimes d)\left(\Delta x^{g_{1}}\right)=\sum_{h \in G} x^{h} \otimes d x^{h^{-1} g_{1}} \\
& \Delta_{R}\left(d x^{g_{1}}\right) \equiv(d \otimes i d)\left(\Delta x^{g_{1}}\right)=\sum_{h \in G} d x^{h} \otimes x^{h^{-1} g_{1}}
\end{aligned}
$$

In the following we will mainly use the pullbacks $\mathcal{L}_{g}$ and $\mathcal{R}_{g}$, rather than the more cumbersome mappings $\Delta_{L}$ and $\Delta_{R}$. The reason we have introduced them is to make

contact with the notations of general Hopf algebras, where the notion of "point on 
the manifold" may not exist. The reader not interested in Hopf algebra formalism can simply ignore the discussions involving $\Delta_{L}$ or $\Delta_{R}$.

A differential calculus is called bicovariant if it is both left and right covariant.

Finally, consider the left action on $e^{g_{1}, g_{2}}=x^{g_{1}} d x^{g_{2}}$ given by eq. (2.30). We see that excluding from the differential algebra the element $e^{g_{1}, g_{2}}$ implies the exclusion of all the elements $e^{h g_{1}, h g_{2}}, h \in G$, that is the exclusion of a subset of $x d x$ elements corresponding to an orbit under left multiplication of the couples $\left(g_{1}, g_{2}\right)$. We call this subset the $e^{g_{1}, g_{2}}$ left orbit. Thus the left-covariant differential calculi on $\operatorname{Fun}(G)$ are obtained from the universal one (where none of the $e^{g_{1}, g_{2}}$ is excluded) by excluding one or more $e^{g_{1}, g_{2}}$ left orbits [5]. Analogous considerations hold for right-invariant calculi.

\subsection{Left and right-invariant one-forms}

As for Lie group manifolds, also in the case of finite groups one can construct left and right invariant one-forms, which provide a basis ("vielbein basis" or cotangent basis) for the vector space $\Gamma$ of one-forms. Following the usual definition, leftinvariant one forms $\theta$ are elements of $\Gamma$ satisfying:

$$
\mathcal{L}_{g} \theta=\theta
$$

In terms of the $\Delta_{L}$ mapping this means:

$$
\Delta_{L} \theta=I \otimes \theta
$$

(use 2.32 and $I(g)=1$ ). It is a simple matter, via eq. (2.28), to show that the one-forms:

$$
\theta^{g} \equiv \sum_{h \in G} x^{h g} d x^{h} \quad\left(=\sum_{h \in G} x^{h} d x^{h g^{-1}}\right),
$$

are indeed left-invariant: $\Delta_{L} \theta^{g}=I \otimes \theta^{g}$, or equivalently : $\mathcal{L}_{h} \theta^{g}=\theta^{g}$.

The relations (2.37) can be inverted :

$$
d x^{h}=\sum_{g \in G}\left(x^{h g}-x^{h}\right) \theta^{g}
$$

From $\sum_{g \in G} d x^{g}=0$ one finds:

$$
\sum_{g \in G} \theta^{g}=\sum_{g \in G} \sum_{h \in G} x^{h} d x^{h g^{-1}}=\sum_{h \in G} x^{h} \sum_{g \in G} d x^{h g^{-1}}=0
$$

We can take as basis of the cotangent space $\Gamma$ the $n-1$ linearly independent left-invariant one-forms $\theta^{g}$ with $g \neq e$. This basis corresponds to the "universal" differential calculus [5]. Smaller sets of $\theta^{g}$ can be chosen as basis (see below).

Notice that in the definition of $\theta^{g}$ the whole $e^{g, e}$ orbit is involved, cf. (2.37). Thus the left-invariant one-forms are in 1-1 correspondence with the (g,e) left orbits: 
removing the $e^{g, e}$ left orbit means to remove $\theta^{g}$. All left-covariant differential calculi are therefore obtained by excluding (i.e. setting to zero) some of the $\theta^{g}$.

The remaining $\theta^{g}(g \neq e)$ constitute a basis for the bimodule $\Gamma$. The $x, \theta$ commutations (bimodule relations) are easily found:

$$
x^{h} d x^{g}=x^{h} \theta^{g^{-1} h}=\theta^{g^{-1} h} x^{g} \quad(h \neq g) \quad \Rightarrow x^{h} \theta^{g}=\theta^{g} x^{h g^{-1}} \quad(g \neq e)
$$

implying the general commutation rule between functions and left-invariant oneforms:

$$
f \theta^{g}=\theta^{g} \mathcal{R}_{g} f
$$

Thus functions do commute between themselves (i.e. $\operatorname{Fun}(G)$ is a commutative algebra) but do not commute with the basis of one-forms $\theta^{g}$. In this sense the differential geometry of $\operatorname{Fun}(G)$ is noncommutative, the noncommutativity being milder than in the case of quantum groups $F u n_{q}(G)$ (which are noncommutative algebras).

Analogous results hold for right invariant one-forms $\omega^{g}$, the corresponding formulae being:

$$
\begin{gathered}
\omega^{g}=\sum_{h \in G} x^{g h} d x^{h}, \quad \Delta_{R} \omega^{g}=\omega^{g} \otimes I \\
f \omega^{g}=\omega^{g} \mathcal{L}_{g} f
\end{gathered}
$$

From the expressions of $\theta^{g}$ and $\omega^{g}$ in terms of $x d x$, one finds the relations

$$
\theta^{g}=\sum_{h \in G} x^{h} \omega^{a d(h) g}, \quad \omega^{g}=\sum_{h \in G} x^{h} \theta^{a d\left(h^{-1}\right) g}
$$

For a bicovariant calculus the right action on $\theta^{g}$ is given by (use the definitions of $\Delta_{R}$ and of $\left.\theta^{g}\right)$ :

$$
\Delta_{R} \theta^{g}=\sum_{h \in G} \theta^{a d(h) g} \otimes x^{h}, \text { or } \mathcal{R}_{h} \theta^{g}=\theta^{a d(h) g}
$$

where $a d$ is the adjoint action of $G$ on $G$, i.e. $a d(h) g \equiv h g h^{-1}$. Then bicovariant calculi are in 1-1 correspondence with unions of conjugacy classes (different from $\{e\})$ [5]: if $\theta^{g}$ is set to zero, one must set to zero all the $\theta^{\operatorname{ad}(h) g}, \forall h \in G$ corresponding to the whole conjugation class of $g$.

As in [5] we denote by $G^{\prime}$ the subset corresponding to the union of conjugacy classes that characterizes the bicovariant calculus on $G\left(G^{\prime}=\left\{g \in G \mid \theta^{g} \neq 0\right\}\right)$. Unless otherwise indicated, hereafter repeated indices are summed on $G^{\prime}$.

A bi-invariant (i.e. left and right invariant) one-form $\Theta$ is obtained by summing on all $\theta^{g}$ or $\omega^{g}$ with $g \neq e$ :

$$
\Theta=\sum_{g \neq e} \theta^{g}=\sum_{g \neq e} \omega^{g}
$$

Note 2.4.1: since $\sum_{g \in G} \theta^{g}=0=\sum_{g \in G} \omega^{g}$, cf. (2.39), we have also $\theta^{e}=-\Theta=$ $\omega^{e}$. 


\subsection{Exterior product}

For a bicovariant differential calculus on a Hopf algebra $A$ an exterior product, compatible with the left and right actions of $G$, can be defined by means of a bimodule automorphism $\Lambda$ in $\Gamma \otimes \Gamma$ that generalizes the ordinary permutation operator:

$$
\Lambda(\theta \otimes \omega)=\omega \otimes \theta
$$

where $\theta$ and $\omega$ are respectively left and right invariant elements of $\Gamma[7]$. Bimodule automorphism means that

$$
\begin{aligned}
& \Lambda(a \eta)=a \Lambda(\eta) \\
& \Lambda(\eta b)=\Lambda(\eta) b
\end{aligned}
$$

for any $\eta \in \Gamma \otimes \Gamma$ and $a, b \in A$. The tensor product between elements $\rho, \rho^{\prime} \in \Gamma$ is defined to have the properties $\rho a \otimes \rho^{\prime}=\rho \otimes a \rho^{\prime}, a\left(\rho \otimes \rho^{\prime}\right)=(a \rho) \otimes \rho^{\prime}$ and $\left(\rho \otimes \rho^{\prime}\right) a=\rho \otimes\left(\rho^{\prime} a\right)$.

Left and right actions on $\Gamma \otimes \Gamma$ are defined by $円$ :

$$
\begin{aligned}
& \Delta_{L}\left(\rho \otimes \rho^{\prime}\right) \equiv \rho_{1} \rho_{1}^{\prime} \otimes \rho_{2} \otimes \rho_{2}^{\prime}, \quad \Delta_{L}: \Gamma \otimes \Gamma \rightarrow A \otimes \Gamma \otimes \Gamma \\
& \Delta_{R}\left(\rho \otimes \rho^{\prime}\right) \equiv \rho_{1} \otimes \rho_{1}^{\prime} \otimes \rho_{2} \rho_{2}^{\prime}, \quad \Delta_{R}: \Gamma \otimes \Gamma \rightarrow \Gamma \otimes \Gamma \otimes A
\end{aligned}
$$

where $\rho_{1}, \rho_{2}$, etc., are a customary short-hand notation defined by

$$
\begin{gathered}
\Delta_{L}(\rho)=\rho_{1} \otimes \rho_{2}, \quad \rho_{1} \in A, \rho_{2} \in \Gamma \\
\Delta_{R}(\rho)=\rho_{1} \otimes \rho_{2}, \quad \rho_{1} \in \Gamma, \rho_{2} \in A .
\end{gathered}
$$

Left-invariance on $\Gamma \otimes \Gamma$ is naturally defined as $\Delta_{L}\left(\rho \otimes \rho^{\prime}\right)=I \otimes \rho \otimes \rho^{\prime}$ (similar definition for right-invariance), so that, for example, $\theta^{i} \otimes \theta^{j}$ is left-invariant, and is in fact a left-invariant basis for $\Gamma \otimes \Gamma$ if $\left\{\theta^{i}\right\}$ is a left-invariant basis for $\Gamma$.

The definition of $\mathcal{L}_{g}$ and $\mathcal{R}_{g}$ on tensor products $\Gamma \otimes \ldots \otimes \Gamma$ is straightforward; for example:

$$
\begin{gathered}
\mathcal{L}_{g}\left(\rho \otimes \rho^{\prime}\right) \equiv \Delta_{L}\left(\rho \otimes \rho^{\prime}\right)[g]=\rho_{1} \rho_{1}^{\prime}(g) \rho_{2} \otimes \rho_{2}^{\prime}=\mathcal{L}_{g} \rho \otimes \mathcal{L}_{g} \rho^{\prime} \\
\mathcal{R}_{g}\left(\rho \otimes \rho^{\prime}\right) \equiv \Delta_{R}\left(\rho \otimes \rho^{\prime}\right)[g]=\rho_{1} \otimes \rho_{1}^{\prime} \rho_{2} \rho_{2}^{\prime}(g)=\mathcal{R}_{g} \rho \otimes \mathcal{R}_{g} \rho^{\prime}
\end{gathered}
$$

where the last equality in both equations is derived after expanding the generic form $\rho$ on the $\theta^{i}$ basis $\left(\rho=f_{i} \theta^{i}\right)$ and likewise for $\rho^{\prime}$. In particular $\mathcal{L}_{h}\left(\theta^{i} \otimes \theta^{j}\right)=\theta^{i} \otimes \theta^{j}$, $\mathcal{R}_{h}\left(\theta^{i} \otimes \theta^{j}\right)=\theta^{a d(h) i} \otimes \theta^{a d(h) j}$.

\footnotetext{
${ }^{1}$ More generally, we can define the action of $\Delta_{L}$ on $\Gamma \otimes \Gamma \otimes \cdots \otimes \Gamma$ as

$$
\begin{gathered}
\Delta_{L}\left(\rho \otimes \rho^{\prime} \otimes \cdots \otimes \rho^{\prime \prime}\right) \equiv \rho_{1} \rho_{1}^{\prime} \cdots \rho_{1}^{\prime \prime} \otimes \rho_{2} \otimes \rho_{2}^{\prime} \otimes \cdots \otimes \rho_{2}^{\prime \prime} \\
\Delta_{L}: \Gamma \otimes \Gamma \otimes \cdots \otimes \Gamma \rightarrow A \otimes \Gamma \otimes \Gamma \otimes \cdots \otimes \Gamma \\
\Delta_{R}\left(\rho \otimes \rho^{\prime} \otimes \cdots \otimes \rho^{\prime \prime}\right) \equiv \rho_{1} \otimes \rho_{1}^{\prime} \cdots \otimes \rho_{1}^{\prime \prime} \otimes \rho_{2} \rho_{2}^{\prime} \cdots \rho_{2}^{\prime \prime} \\
\Delta_{R}: \Gamma \otimes \Gamma \otimes \cdots \otimes \Gamma \rightarrow \Gamma \otimes \Gamma \otimes \cdots \otimes \Gamma \otimes A .
\end{gathered}
$$
}


Note 2.5.1: In general $\Lambda^{2} \neq 1$, since $\Lambda\left(\omega^{j} \otimes \theta^{i}\right)$ is not necessarily equal to $\theta^{i} \otimes \omega^{j}$. By linearity, $\Lambda$ can be extended to the whole of $\Gamma \otimes \Gamma$.

Note 2.5.2: $\Lambda$ is invertible and commutes with the left and right action of $G$, i.e. $\Delta_{L} \Lambda\left(\rho \otimes \rho^{\prime}\right)=(i d \otimes \Lambda) \Delta_{L}\left(\rho \otimes \rho^{\prime}\right)=\rho_{1} \rho_{1}^{\prime} \otimes \Lambda\left(\rho_{2} \otimes \rho_{2}^{\prime}\right)$, and similar for $\Delta_{R}$. Equivalently: $\mathcal{L}_{g} \Lambda\left(\rho \otimes \rho^{\prime}\right)=\Lambda\left[\mathcal{L}_{g}\left(\rho \otimes \rho^{\prime}\right)\right]=\Lambda\left(\mathcal{L}_{g} \rho \otimes \mathcal{L}_{g} \rho^{\prime}\right)$ and similar for $\mathcal{R}_{g}$. Therefore $\Lambda\left(\theta^{i} \otimes \theta^{j}\right)$ is left-invariant, and can be expanded on the left-invariant basis $\theta^{i} \otimes \theta^{j}$ :

$$
\Lambda\left(\theta^{i} \otimes \theta^{j}\right)=\Lambda_{k l}^{i j} \theta^{k} \otimes \theta^{l}
$$

The exterior product is defined as:

$$
\begin{aligned}
& \rho \wedge \rho^{\prime} \equiv \rho \otimes \rho^{\prime}-\Lambda\left(\rho \otimes \rho^{\prime}\right) \\
& \theta^{i} \wedge \theta^{j} \equiv W_{k l}^{i j} \theta^{k} \otimes \theta^{l}=\theta^{i} \otimes \theta^{j}-\Lambda_{k l}^{i j} \theta^{k} \otimes \theta^{l} .
\end{aligned}
$$

where $\rho, \rho^{\prime} \in \Gamma$ and $\left\{\theta^{i}\right\}=$ left-invariant basis for $\Gamma$. Notice that, given the matrix $\Lambda_{k l}^{i j}$, we can compute the exterior product of any $\rho, \rho^{\prime} \in \Gamma$, since any $\rho \in \Gamma$ is expressible in terms of $\theta^{i}$.

In the case $A=\operatorname{Fun}(G)$, we find

$$
\begin{aligned}
\Lambda\left(\theta^{g} \otimes \theta^{g^{\prime}}\right) & =\Lambda\left(\theta^{g} \otimes \sum_{h \in G} x^{h} \omega^{a d(h) g^{\prime}}\right)=\sum_{h \in G} x^{h g} \Lambda\left(\theta^{g} \otimes \omega^{a d(h) g^{\prime}}\right)= \\
& =\sum_{h \in G} x^{h g} \omega^{a d(h) g^{\prime}} \otimes \theta^{g}=\sum_{h, h^{\prime} \in G} x^{h g} x^{h^{\prime}} \theta^{a d\left(h^{\prime-1} h\right) g^{\prime}} \otimes \theta^{g}= \\
& =\sum_{h \in G} x^{h g} \theta^{a d\left(g^{-1}\right) g^{\prime}} \otimes \theta^{g}=\theta^{a d\left(g^{-1}\right) g^{\prime}} \otimes \theta^{g}
\end{aligned}
$$

and the $\Lambda_{k l}^{i j}$ matrix takes the form:

$$
\Lambda_{h_{1}, h_{2}}^{g_{1}, g_{2}}=\delta_{h_{2}}^{g_{1}} \delta_{h_{1}}^{a d\left(g_{1}^{-1}\right) g_{2}}
$$

Then the exterior product of two left-invariant basic one-forms is given by:

$$
\theta^{g_{1}} \wedge \theta^{g_{2}}=\theta^{g_{1}} \otimes \theta^{g_{2}}-\theta^{g_{1}^{-1} g_{2} g_{1}} \otimes \theta^{g_{1}}
$$

Note that:

$$
\theta^{g} \wedge \theta^{g}=0 \quad(\text { no sum on } g)
$$

This familiar formula holds for $F u n(G)$, but not for a general Hopf algebra.

We can generalize the definition (2.58) to exterior products of $n$ one-forms:

$$
\theta^{i_{1}} \wedge \ldots \wedge \theta^{i_{n}} \equiv W_{j_{1 . . j}}^{i_{1} . . i_{n}} \theta^{j_{1}} \otimes \ldots \otimes \theta^{j_{n}}
$$

or in short-hand notation:

$$
\theta^{1} \wedge \ldots \wedge \theta^{n}=W_{1 . . n} \theta^{1} \otimes \ldots \otimes \theta^{n}
$$


where the labels $1 . . n$ in $W$ refer to index couples. The numerical coefficients $W_{1 \ldots n}$ are given through a recursion relation

$$
W_{1 \ldots n}=\mathcal{I}_{1 \ldots n} W_{1 \ldots n-1}
$$

where

$$
\mathcal{I}_{1 \ldots n}=1-\Lambda_{n-1, n}+\Lambda_{n-2, n-1} \Lambda_{n-1, n} \ldots-(-1)^{n} \Lambda_{12} \Lambda_{23} \cdots \Lambda_{n-1, n}
$$

and $W_{1}=1$. The space of $n$-forms $\Gamma^{\wedge n}$ is therefore defined as in the usual case but with the new permutation operator $\Lambda$, and can be shown to be a bicovariant bimodule (see for ex. [10]), with left and right action defined as for $\Gamma \otimes \ldots \otimes \Gamma$ with the tensor product replaced by the wedge product.

\subsection{Exterior derivative}

With the exterior product we can define the exterior derivative

$$
\begin{gathered}
d: \Gamma \rightarrow \Gamma \wedge \Gamma \\
d\left(a_{k} d b_{k}\right)=d a_{k} \wedge d b_{k},
\end{gathered}
$$

which can easily be extended to $\Gamma^{\wedge n}\left(d: \Gamma^{\wedge n} \rightarrow \Gamma^{\wedge(n+1)}\right)$, and has the following properties:

$$
\begin{gathered}
d\left(\rho \wedge \rho^{\prime}\right)=d \rho \wedge \rho^{\prime}+(-1)^{k} \rho \wedge d \rho^{\prime} \\
d(d \rho)=0 \\
\Delta_{L}(d \rho)=(i d \otimes d) \Delta_{L}(\rho) \text { or } \quad \mathcal{L}_{g}(d \rho)=d\left(\mathcal{L}_{g} \rho\right) \\
\Delta_{R}(d \rho)=(d \otimes i d) \Delta_{R}(\rho) \text { or } \mathcal{R}_{g}(d \rho)=d\left(\mathcal{R}_{g} \rho\right),
\end{gathered}
$$

where $\rho \in \Gamma^{\wedge k}, \rho^{\prime} \in \Gamma^{\wedge n}, \Gamma^{\wedge 0} \equiv F u n(G)$. The last two properties show that $d$ commutes with the left and right action of $G$.

\subsection{Tangent vectors}

In (2.21) we expressed $d f$ in terms of the differentials $d x^{g}$. Using (2.38) we find the expansion of $d f$ on the basis of the left-invariant one-forms $\theta^{g}$ :

$$
d f=\sum_{g \in G} f_{g} d x^{g}=\sum_{g \in G} f_{g} \sum_{h \in G^{\prime}}\left(x^{g h}-x^{g}\right) \theta^{h}=\sum_{h \in G^{\prime}}\left(\mathcal{R}_{h^{-1}} f-f\right) \theta^{h} \equiv \sum_{h \in G^{\prime}}\left(t_{h} f\right) \theta^{h}
$$

so that the "flat" partial derivatives $t_{h} f$ are given by

$$
t_{h} f=\mathcal{R}_{h^{-1}} f-f
$$

Note that $t_{h} f$ are really functions $\in F u n(G)$, whereas the "curved" partial derivatives of eq. (2.22) are numbers. The Leibniz rule for the flat partial derivatives $t_{g}$ reads:

$$
t_{g}\left(f f^{\prime}\right)=\left(t_{g} f\right) \mathcal{R}_{g^{-1}} f^{\prime}+f t_{g} f^{\prime}
$$


In analogy with ordinary differential calculus, the operators $t_{g}$ appearing in (2.73) are called (left-invariant) tangent vectors, and in our case are given by

$$
t_{g}=\mathcal{R}_{g^{-1}}-i d
$$

They satisfy the composition rule:

$$
t_{g} t_{g^{\prime}}=\sum_{h} C_{g, g^{\prime}}^{h} t_{h}
$$

where the structure constants are:

$$
C_{g, g^{\prime}}^{h}=\delta_{g^{\prime} g}^{h}-\delta_{g}^{h}-\delta_{g^{\prime}}^{h}
$$

and have the property:

$$
C^{a d(h) g_{1}} \underset{a d(h) g_{2}, a d(h) g_{3}}{a}=C_{g_{2}, g_{3}}^{g_{1}}
$$

Clearly we can expand $d f$ also on the right-invariant basis $\omega^{g}$ and define (rightinvariant) tangent vectors $\tilde{t}_{h}$ from $d f=\sum_{h}\left(\tilde{t}_{h} f\right) \omega^{h}$, whose explicit operator expression is:

$$
\tilde{t}_{g}=\mathcal{L}_{g^{-1}}-i d
$$

Note 2.7.1 : The exterior derivative on any $f \in F u n(G)$ can be expressed as a commutator of $f$ with the bi-invariant one-form $\Theta$ :

$$
d f=[\Theta, f]
$$

as one proves by using (2.41) and (2.73).

Note 2.7.2 : From the fusion rules (2.77) we deduce the "deformed Lie algebra" (cf. ref.s [7, 8, 9, 10]):

$$
t_{g_{1}} t_{g_{2}}-\Lambda_{g_{3}, g_{4}, g_{2}}^{g_{g_{3}}} t_{g_{3}} t_{g_{4}}=C^{h}{ }_{g_{1}, g_{2}} t_{h}
$$

where the $\boldsymbol{C}$ structure constants are given by:

$$
C^{g}{ }_{g_{1}, g_{2}} \equiv C^{g}{ }_{g_{1}, g_{2}}-\Lambda_{g_{1}, g_{2}}^{g_{3}, g_{4}} C_{g_{3}, g_{4}}^{g}=C^{g}{ }_{g_{1}, g_{2}}-C^{g}{ }_{g_{2}, g_{2} g_{1} g_{2}^{-1}}=\delta_{g_{1}}^{a d\left(g_{2}^{-1}\right) g}-\delta_{g_{1}}^{g}
$$

and besides property (2.79) they also satisfy:

$$
C_{g_{1}, g_{2}}^{g}=\boldsymbol{C}_{g, g_{2}^{-1}}^{g_{1}}
$$

Moreover the following identities hold:

i) deformed Jacobi identities:

$$
C^{k}{ }_{h_{1}, g_{1}} C^{h_{2}}{ }_{k, g_{2}}-\Lambda^{g_{3}, g_{1}}{ }_{g_{1}, g_{2}} C^{k}{ }_{h_{1}, g_{3}} C^{h_{2}}{ }_{k, g_{4}}=C^{k}{ }_{g_{1}, g_{2}} C^{h_{2}}{ }_{h_{1}, k}
$$

ii) fusion identities:

$$
\boldsymbol{C}_{h_{1}, g}^{k} \boldsymbol{C}_{k, g^{\prime}}^{h_{2}}=C_{g, g^{\prime}}^{h} \boldsymbol{C}_{h_{1}, h}^{h_{2}}
$$

Thus the $\boldsymbol{C}$ structure constants are a representation (the adjoint representation) of the tangent vectors $t$.

Note 2.7.3: The fusion rules (2.77) also allow to associate an ordinary (i.e. not deformed) Lie algebra to the finite group $G$; the corresponding structure constants are simply twice the antisymmetric part (in the indices $g_{1}, g_{2}$ ) of $C_{g_{1}, g_{2}}^{g}$. 


\subsection{Cartan-Maurer equations, connection and curvature}

From the definition (2.37) and eq. (2.40) we deduce the Cartan-Maurer equations:

$$
d \theta^{g}+\sum_{g_{1}, g_{2}} C^{g}{ }_{g_{1}, g_{2}} \theta^{g_{1}} \wedge \theta^{g_{2}}=0
$$

where the structure constants $C^{g}{ }_{g_{1}, g_{2}}$ are those given in (2.78).

Parallel transport of the vielbein $\theta^{g}$ can be defined as in ordinary Lie group manifolds:

$$
\nabla \theta^{g}=-\omega_{g^{\prime}}^{g} \otimes \theta^{g^{\prime}}
$$

where $\omega_{g_{2}}^{g_{1}}$ is the connection one-form:

$$
\omega_{g_{2}}^{g_{1}}=\Gamma_{g_{3}, g_{2}}^{g_{1}} \theta^{g_{3}}
$$

Thus parallel transport is a map from $\Gamma$ to $\Gamma \otimes \Gamma$; by definition it must satisfy:

$$
\nabla(a \rho)=(d a) \otimes \rho+a \nabla \rho, \quad \forall a \in A, \rho \in \Gamma
$$

and it is a simple matter to verify that this relation is satisfied with the usual parallel transport of Riemannian manifolds. As for the exterior differential, $\nabla$ can be extended to a map $\nabla: \Gamma^{\wedge n} \otimes \Gamma \longrightarrow \Gamma^{\wedge(n+1)} \otimes \Gamma$ by defining:

$$
\nabla(\varphi \otimes \rho)=d \varphi \otimes \rho+(-1)^{n} \varphi \nabla \rho
$$

Requiring parallel transport to commute with the left and right action of $G$ means:

$$
\begin{aligned}
& \mathcal{L}_{h}\left(\nabla \theta^{g}\right)=\nabla\left(\mathcal{L}_{h} \theta^{g}\right)=\nabla \theta^{g} \\
& \mathcal{R}_{h}\left(\nabla \theta^{g}\right)=\nabla\left(\mathcal{R}_{h} \theta^{g}\right)=\nabla \theta^{a d(h) g}
\end{aligned}
$$

Recalling that $\mathcal{L}_{h}(a \rho)=\left(\mathcal{L}_{h} a\right)\left(\mathcal{L}_{h} \rho\right)$ and $\mathcal{L}_{h}\left(\rho \otimes \rho^{\prime}\right)=\left(\mathcal{L}_{h} \rho\right) \otimes\left(\mathcal{L}_{h} \rho^{\prime}\right), \quad \forall a \in$ $A, \rho, \rho^{\prime} \in \Gamma$ (and similar for $\mathcal{R}_{h}$ ), and substituting (2.88) yields respectively:

$$
\Gamma_{g_{3}, g_{2}}^{g_{1}} \in \boldsymbol{C}
$$

and

$$
\Gamma_{\quad a d(h) g_{3}, a d(h) g_{2}}^{a d(h) g_{1}}=\Gamma_{g_{3}, g_{2}}^{g_{1}}
$$

Therefore the same situation arises as in the case of Lie groups, for which parallel transport on the group manifold commutes with left and right action iff the connection components are $a d(G)$ - conserved constant tensors. As for Lie groups, condition (2.95) is satisfied if one takes $\Gamma$ proportional to the structure constants. In our case, we can take any combination of the $C$ or $\boldsymbol{C}$ structure constants, since both are $\operatorname{ad}(G)$ conserved constant tensors. As we see below, the $C$ constants can be used to define a torsionless connection, while the $\boldsymbol{C}$ constants define a parallelizing connection. 
As usual, the curvature arises from $\nabla^{2}$ :

$$
\begin{gathered}
\nabla^{2} \theta^{g}=-R_{g^{\prime}}^{g} \otimes \theta^{g^{\prime}} \\
R_{g_{2}}^{g_{1}} \equiv d \omega_{g_{2}}^{g_{1}}+\omega_{g_{3}}^{g_{1}} \wedge \omega_{g_{2}}^{g_{3}}
\end{gathered}
$$

The torsion $R^{g}$ is defined by:

$$
R^{g_{1}} \equiv d \theta^{g_{1}}+\omega_{g_{2}}^{g_{1}} \wedge \theta^{g_{2}}
$$

Using the expression of $\omega$ in terms of $\Gamma$ and the Cartan-Maurer equations yields

$$
\begin{aligned}
R_{g_{2}}^{g_{1}}= & \left(-\Gamma_{h, g_{2}}^{g_{1}} C_{g_{3}, g_{4}}^{h}+\Gamma_{g_{3}, h}^{g_{1}} \Gamma_{g_{4}, g_{2}}^{h}\right) \theta^{g_{3}} \wedge \theta^{g_{4}}= \\
= & \left(-\Gamma_{h, g_{2}}^{g_{1}} C_{g_{3}, g_{4}}^{h}+\Gamma_{g_{3}, h}^{g_{1}} \Gamma_{g_{4}, g_{2}}^{h}-\Gamma_{g_{4}, h}^{g_{1}} \Gamma_{g_{4} g_{3} g_{4}^{-1}, g_{2}}^{h}\right) \theta^{g_{3}} \otimes \theta^{g_{4}} \\
& R^{g_{1}}=\left(-C_{g_{2}, g_{3}}^{g_{1}}+\Gamma_{g_{2}, g_{3}}^{g_{1}}\right) \theta^{g_{2}} \wedge \theta^{g_{3}}= \\
& =\left(-C_{g_{2}, g_{3}}^{g_{1}}+\Gamma_{g_{2}, g_{3}}^{g_{1}}-\Gamma_{g_{3}, g_{3} g_{2} g_{3}^{-1}}^{g_{1}}\right) \theta^{g_{2}} \otimes \theta^{g_{3}}
\end{aligned}
$$

Thus a connection satisfying:

$$
\Gamma_{g_{2}, g_{3}}^{g_{1}}-\Gamma_{g_{3}, g_{3} g_{2} g_{3}^{-1}}^{g_{1}}=\boldsymbol{C}_{g_{2}, g_{3}}^{g_{1}}
$$

corresponds to a vanishing torsion $R^{g}=0$ and could be referred to as a "Riemannian" connection.

On the other hand, the choice:

$$
\Gamma_{g_{2}, g_{3}}^{g_{1}}=\boldsymbol{C}_{g_{3}, g_{2}^{-1}}^{g_{1}}
$$

corresponds to a vanishing curvature $R_{g^{\prime}}^{g}=0$, as can be checked by using the fusion equations (2.86) and property (2.84). Then (2.102) can be called the parallelizing connection: finite groups are parallelizable.

\subsection{Tensor transformations and covariant derivative}

Under the familiar transformation of the connection 1-form:

$$
\left(\omega_{j}^{i}\right)^{\prime}=a_{k}^{i} \omega^{k}{ }_{l}\left(a^{-1}\right)^{l}{ }_{j}+a_{k}^{i} d\left(a^{-1}\right)^{k}{ }_{j}
$$

the curvature 2-form transforms homogeneously:

$$
\left(R^{i}{ }_{j}\right)^{\prime}=a_{k}^{i} R_{l}^{k}\left(a^{-1}\right)^{l}{ }_{j}
$$

The transformation rule (2.103) can be seen as induced by the change of basis $\theta^{i}=a^{i}{ }_{j} \theta^{j}$, with $a^{i}{ }_{j}$ invertible $x$-dependent matrix (use eq. (2.90) with $a \rho=a_{j}^{i} \theta^{j}$ ). 
The covariant derivative $D$ of a function $\phi_{i}$ transforming as $\phi_{i}^{\prime}=\phi_{j}\left(a^{-1}\right)^{j}{ }_{i}(i=$ contravariant index) is defined as follows:

$$
D \phi_{i} \equiv d \phi_{i}-\phi_{j} \omega_{i}^{j}
$$

(or equivalently by $\nabla \phi \equiv D \phi_{i} \otimes \theta^{i}$, with $\phi=\phi_{i} \theta^{i}$ ), and indeed transforms homogeneously $\left(D \phi_{i}\right)^{\prime}=\left(D \phi_{j}\right)\left(a^{-1}\right)^{j}{ }_{i}$

Similarly on a function $\varphi^{i}$ with a covariant index transforming as $\left(\varphi^{i}\right)^{\prime}=a^{i}{ }_{j} \varphi^{j}$ the covariant derivative is:

$$
D \varphi^{i} \equiv d \varphi^{i}+\omega^{i}{ }_{j} \varphi_{j}
$$

and transforms as $\left(D \varphi^{i}\right)^{\prime}=a^{i}{ }_{j}\left(D \varphi^{j}\right)$. Then $D$ on the scalar $\phi_{i} \varphi^{i}$ reduces to $d$, if one defines $D$ to satisfy the Leibniz rule: $D\left(\phi_{i} \varphi^{i}\right)=\left(D \phi_{i}\right) \varphi^{i}+\phi_{i} D\left(\varphi^{i}\right)$.

The generalization of $D$ on tensors $T$ with an arbitrary number of covariant and contravariant indices is not straightforward, see for example ref. [5, 4] for a discussion. Although a consistent definition of parallel transport for tensors is clearly important, we will not need it in the following.

\subsection{Metric}

The metric tensor $\gamma$ can be defined as an element of $\Gamma \otimes \Gamma$ :

$$
\gamma=\gamma_{i, j} \theta^{i} \otimes \theta^{j}
$$

Requiring it to be invariant under left and right action of $G$ means:

$$
\mathcal{L}_{h}(\gamma)=\gamma=\mathcal{R}_{h}(\gamma)
$$

or equivalently, by recalling $\mathcal{L}_{h}\left(\theta^{i} \otimes \theta^{j}\right)=\theta^{i} \otimes \theta^{j}, \mathcal{R}_{h}\left(\theta^{i} \otimes \theta^{j}\right)=\theta^{a d(h) i} \otimes \theta^{a d(h) j}$ :

$$
\gamma_{i, j} \in \boldsymbol{C}, \quad \gamma_{a d(h) i, a d(h) j}=\gamma_{i, j}
$$

These properties are analogous to the ones satisfied by the Killing metric of Lie groups, which is indeed constant and invariant under the adjoint action of the Lie group.

On finite $G$ there are various choices of biinvariant metrics. One can simply take $\gamma_{i, j}=\delta_{i, j}$, or $\gamma_{i, j}=\boldsymbol{C}_{l, i}^{k} \boldsymbol{C}_{k, j}^{l}$, or the "distance" matrix defined in Section 3 . Note that we are not insisting here on a covariantly conserved metric (i.e. a metric compatible connection, see ref. ([5])).

For any biinvariant metric $\gamma_{i, j}$ there are tensor transformations $a_{j}^{i}$ under which $\gamma_{i, j}$ is invariant, i.e.:

$$
a_{h^{\prime}}^{h} \gamma_{h, k} a_{k^{\prime}}^{k}=\gamma_{h^{\prime}, k^{\prime}} \Leftrightarrow a_{h^{\prime}}^{h} \gamma_{h, k}=\gamma_{h^{\prime}, k^{\prime}}\left(a^{-1}\right)_{k}^{k_{k}^{\prime}}
$$


These transformations are simply given by the matrices that rotate the indices according to the adjoint action of $G$ :

$$
a_{h^{\prime}}^{h}(g)=\delta_{h^{\prime}}^{a d(\alpha(g)) h}
$$

where $\alpha(g): G \mapsto G$ is an arbitrary mapping. Then these matrices are functions of $G$ via this mapping, and their action leaves $\gamma$ invariant because of its biinvariance (2.109). Indeed substituting these matrices in (2.110) yields:

$$
a_{h^{\prime}}^{h}(g) \gamma_{h, k} a_{k^{\prime}}^{k}(g)=\gamma_{a d\left([\alpha(g)]^{-1}\right) h^{\prime}, a d\left([\alpha(g)]^{-1}\right) k^{\prime}}=\gamma_{h^{\prime}, k^{\prime}}
$$

proving the invariance of $\gamma$.

Consider now a contravariant vector $\varphi^{i}$ transforming as $\left(\varphi^{i}\right)^{\prime}=a^{i}{ }_{j}\left(\varphi^{j}\right)$. Then using (2.110) one can easily see that

$$
\left(\varphi^{k} \gamma_{k, i}\right)^{\prime}=\varphi^{k^{\prime}} \gamma_{k^{\prime}, i^{\prime}}\left(a^{-1}\right)^{i^{\prime}}
$$

i.e. the vector $\varphi_{i} \equiv \varphi^{k} \gamma_{k, i}$ indeed transforms as a covariant vector.

\subsection{Lie derivative and diffeomorphisms}

The notion of diffeomorphisms, or general coordinate transformations, is fundamental in gravity theories. Is there such a notion in the setting of differential calculi on Hopf algebras ? The answer is affirmative, and has been discussed in detail in ref.s [8, 9, 10]. As for differentiable manifolds, it relies on the existence of the Lie derivative.

Let us review the situation for Lie group manifolds. The Lie derivative $l_{t_{i}}$ along a left-invariant tangent vector $t_{i}$ is related to the infinitesimal right translations generated by $t_{i}$ :

$$
l_{t_{i}} \rho=\lim _{\varepsilon \rightarrow 0} \frac{1}{\varepsilon}\left[\mathcal{R}_{\exp \left[\varepsilon t_{i}\right]} \rho-\rho\right]
$$

$\rho$ being an arbitrary tensor field. Introducing the coordinate dependence

$$
l_{t_{i}} \rho(y)=\lim _{\varepsilon \rightarrow 0} \frac{1}{\varepsilon}\left[\rho\left(y+\varepsilon t_{i}\right)-\rho(y)\right]
$$

identifies the Lie derivative $l_{t_{i}}$ as a directional derivative along $t_{i}$. Note the difference in meaning of the symbol $t_{i}$ in the r.h.s. of these two equations: a group generator in the first, and the corresponding tangent vector in the second.

To find the natural generalization of the Lie derivative in the case of finite groups, we express formula (2.114) in a completely algebraic notation:

$$
l_{t_{i}} \rho=\left(i d \otimes t_{i}\right) \Delta_{R}(\rho)
$$

This expression is well defined for any Hopf algebra. In particular for finite groups (2.116) takes the form:

$$
l_{t_{g}} \rho=\left[\mathcal{R}_{g^{-1}} \rho-\rho\right]
$$


so that the Lie derivative is simply given by

$$
l_{t_{g}}=\mathcal{R}_{g^{-1}}-i d=t_{g}
$$

cf. the definition of $t_{g}$ in (2.76). For example

$$
l_{t_{g}}\left(\theta^{g_{1}} \otimes \theta^{g_{2}}\right)=\theta^{a d\left(g^{-1}\right) g_{1}} \otimes \theta^{a d\left(g^{-1}\right) g_{2}}-\theta^{g_{1}} \otimes \theta^{g_{2}}
$$

As in the case of differentiable manifolds, the Cartan formula for the Lie derivative acting on p-forms holds:

$$
l_{t_{g}}=i_{t_{g}} d+d i_{t_{g}}
$$

(see Appendix A ).

Exploiting this formula, diffeomorphisms (Lie derivatives) along generic tangent vectors $V$ can also be consistently defined via the operator:

$$
l_{V}=i_{V} d+d i_{V}
$$

This requires a suitable definition of the contraction operator $i_{V}$ along generic tangent vectors $V$, discussed in Appendix A.

We have then a way of defining "diffeomorphisms" along arbitrary (and xdependent) tangent vectors for any tensor $\rho$ :

$$
\delta \rho=l_{V} \rho
$$

and of testing the invariance of candidate lagrangians under the generalized Lie derivative.

\subsection{Haar measure and integration}

Since we want to be able to define actions (integrals on $p$-forms) we must now define integration of $p$-forms on finite groups.

Let us start with integration of functions $f$. We define the integral map $h$ as a linear functional $h: F u n(G) \mapsto C$ satisfying the left and right invariance conditions:

$$
h\left(\mathcal{L}_{g} f\right)=0=h\left(\mathcal{R}_{g} f\right)
$$

Then this map is uniquely determined (up to a normalization constant), and is simply given by the "sum over $G$ " rule:

$$
h(f)=\sum_{g \in G} f(g)
$$

Next we turn to define the integral of a p-form. Within the differential calculus we have a basis of left-invariant 1-forms, which may allow the definition of a biinvariant volume element. In general for a differential calculus with $n$ independent tangent vectors, there is an integer $p \geq n$ such that the linear space of $p$-forms is 1-dimensional, and $(p+1)$ - forms vanish identically. We will see explicit 
examples in the next Section. This means that every product of $p$ basis one-forms $\theta^{g_{1}} \wedge \theta^{g_{2}} \wedge \ldots \wedge \theta^{g_{p}}$ is proportional to one of these products, that can be chosen to define the volume form $v o l$ :

$$
\theta^{g_{1}} \wedge \theta^{g_{2}} \wedge \ldots \wedge \theta^{g_{p}}=\epsilon^{g_{1}, g_{2}, \ldots g_{p}} \mathrm{vol}
$$

where $\epsilon^{g_{1}, g_{2}, \ldots g_{p}}$ is the proportionality constant. Note that the volume $p$-form is obviously left invariant. We can prove that it is also right invariant with the following argument. Suppose that vol be given by $\theta^{h_{1}} \wedge \theta^{h_{2}} \wedge \ldots \wedge \theta^{h_{p}}$ where $h_{1}, h_{2}, \ldots h_{p}$ are given group element labels. Then the right action on vol yields:

$$
\mathcal{R}_{g}\left[\theta^{h_{1}} \wedge \ldots \wedge \theta^{h_{p}}\right]=\theta^{a d(g) h_{1}} \wedge \ldots \wedge \theta^{a d(g) h_{p}}=\epsilon^{a d(g) h_{1}, \ldots a d(g) h_{p}} \mathrm{vol}
$$

Recall now that the "epsilon tensor" $\epsilon$ is necessarily made out of products of the $\Lambda$ tensor of eq. (2.58), defining the wedge product. This tensor is invariant under the adjoint action $a d(g)$, and so is the $\epsilon$ tensor. Therefore $\epsilon^{a d(g) h_{1}, \ldots a d(g) h_{p}}=\epsilon^{h_{1}, \ldots h_{p}}=1$ and $\mathcal{R}_{g} v o l=v o l$. This will be verified in the examples of next Section.

Having identified the volume $p$-form it is natural to set

$$
\int f v o l \equiv h(f)=\sum_{g \in G} f(g)
$$

and define the integral on a $p$-form $\rho$ as:

$$
\int \rho=\int \rho_{g_{1}, \ldots g_{p}} \theta^{g_{1}} \wedge \ldots \wedge \theta^{g_{p}}=\int \rho_{g_{1}, \ldots g_{p}} \epsilon^{g_{1}, \ldots g_{p}} v o l \equiv \sum_{g \in G} \rho_{g_{1}, \ldots g_{p}}(g) \epsilon^{g_{1}, \ldots g_{p}}
$$

Due to the biinvariance of the volume form, the integral map $\int: \Gamma^{\wedge p} \mapsto \boldsymbol{C}$ satisfies the biinvariance conditions:

$$
\int \mathcal{L}_{g} f=\int f=\int \mathcal{R}_{g} f
$$

Moreover, under the assumption that the volume form belongs to a nontrivial cohomology class, that is $d(v o l)=0$ but $v o l \neq d \rho$, the important property holds:

$$
\int d f=0
$$

with $f$ any $(p-1)$-form: $f=f_{g_{2}, \ldots g_{p}} \theta^{g_{2}} \wedge \ldots \wedge \theta^{g_{p}}$. This property, which allows integration by parts, has a simple proof. Rewrite $\int d f$ as:

$$
\int d f=\int\left(d f_{g_{2}, \ldots g_{p}}\right) \theta^{g_{2}} \wedge \ldots \wedge \theta^{g_{p}}+\int f_{g_{2}, \ldots g_{p}} d\left(\theta^{g_{2}} \wedge \ldots \wedge \theta^{g_{p}}\right)
$$

Under the cohomology assumption the second term in the r.h.s. vanishes, since $d\left(\theta^{g_{2}} \wedge \ldots \wedge \theta^{g_{p}}\right)=0$ (otherwise, being a $p$-form, it should be proportional to $\mathrm{vol}$, and this would contradict the assumption $v o l \neq d \rho$ ). Using now (2.73) and (2.127):

$$
\begin{aligned}
\int d f & =\int\left(t_{g_{1}} f_{g_{2}, \ldots g_{p}}\right) \theta^{g_{1}} \wedge \theta^{g_{2}} \wedge \ldots \wedge \theta^{g_{p}}=\int\left[\mathcal{R}_{g_{1}^{-1}} f_{g_{2}, \ldots g_{p}}-f_{g_{2}, \ldots g_{p}}\right] \epsilon^{g_{1}, \ldots g_{p}} v o l= \\
& =\epsilon^{g_{1}, \ldots g_{p}} \sum_{g \in G}\left[\mathcal{R}_{g_{1}^{-1}} f_{g_{2}, \ldots g_{p}}(g)-f_{g_{2}, \ldots g_{p}}(g)\right]=0
\end{aligned}
$$

Q.E.D. 


\section{Bicovariant calculus on $S_{3}$}

In this Section we illustrate the general theory on the particular example of the permutation group $S_{3}$.

Elements: $a=(12), b=(23), c=(13), a b=(132), b a=(123), e$.

Multiplication table:

\begin{tabular}{|c|c|c|c|c|c|c|}
\hline & e & a & b & c & ab & ba \\
\hline e & e & a & b & c & ab & ba \\
\hline a & a & e & ab & ba & b & c \\
\hline b & b & ba & e & ab & c & a \\
\hline c & c & ab & ba & e & a & b \\
\hline ab & ab & c & a & b & ba & e \\
\hline ba & ba & b & c & a & e & ab \\
\hline
\end{tabular}

Nontrivial conjugation classes: $I=[a, b, c], I I=[a b, b a]$.

There are 3 bicovariant calculi $B C_{I}, B C_{I I}, B C_{I+I I}$ corresponding to the possible unions of the conjugation classes [5]. They have respectively dimension 3,2 and 5 . We examine here the $B C_{I}$ and $B C_{I I}$ calculi.

\section{1 $B C_{I}$ differential calculus}

Basis of the 3-dimensional vector space of one-forms:

$$
\theta^{a}, \theta^{b}, \theta^{c}
$$

Basis of the 4-dimensional vector space of two-forms:

$$
\theta^{a} \wedge \theta^{b}, \theta^{b} \wedge \theta^{c}, \theta^{a} \wedge \theta^{c}, \theta^{c} \wedge \theta^{b}
$$

Every wedge product of two $\theta$ can be expressed as linear combination of the basis elements:

$$
\theta^{b} \wedge \theta^{a}=-\theta^{a} \wedge \theta^{c}-\theta^{c} \wedge \theta^{b}, \quad \theta^{c} \wedge \theta^{a}=-\theta^{a} \wedge \theta^{b}-\theta^{b} \wedge \theta^{c}
$$

Basis of the 3-dimensional vector space of three-forms:

$$
\theta^{a} \wedge \theta^{b} \wedge \theta^{c}, \theta^{a} \wedge \theta^{c} \wedge \theta^{b}, \theta^{b} \wedge \theta^{a} \wedge \theta^{c}
$$

and we have:

$$
\begin{aligned}
& \theta^{c} \wedge \theta^{b} \wedge \theta^{a}=-\theta^{c} \wedge \theta^{a} \wedge \theta^{c}=-\theta^{a} \wedge \theta^{c} \wedge \theta^{a}=\theta^{a} \wedge \theta^{b} \wedge \theta^{c} \\
& \theta^{b} \wedge \theta^{c} \wedge \theta^{a}=-\theta^{b} \wedge \theta^{a} \wedge \theta^{b}=-\theta^{a} \wedge \theta^{b} \wedge \theta^{a}=\theta^{a} \wedge \theta^{c} \wedge \theta^{b} \\
& \theta^{c} \wedge \theta^{a} \wedge \theta^{b}=-\theta^{c} \wedge \theta^{b} \wedge \theta^{c}=-\theta^{b} \wedge \theta^{c} \wedge \theta^{b}=\theta^{b} \wedge \theta^{a} \wedge \theta^{c}
\end{aligned}
$$


Basis of the 1-dimensional vector space of four-forms:

$$
\text { vol }=\theta^{a} \wedge \theta^{b} \wedge \theta^{a} \wedge \theta^{c}
$$

and we have:

$$
\theta^{g_{1}} \wedge \theta^{g_{2}} \wedge \theta^{g_{3}} \wedge \theta^{g_{4}}=\epsilon^{g_{1}, g_{2}, g_{3}, g_{4}} \text { vol }
$$

where the nonvanishing components of the $\epsilon$ tensor are:

$$
\begin{aligned}
& \epsilon_{a b a c}=\epsilon_{a c a b}=\epsilon_{c b c a}=\epsilon_{c a c b}=\epsilon_{b a b c}=\epsilon_{b c b a}=1 \\
& \epsilon_{b a c a}=\epsilon_{c a b a}=\epsilon_{a b c b}=\epsilon_{c b a b}=\epsilon_{a c b c}=\epsilon_{b c a c}=-1
\end{aligned}
$$

Note the interesting property

$$
f \text { vol }=\operatorname{vol} f, \quad \forall f \in F u n(G)
$$

due to $\mathcal{R}_{a} \mathcal{R}_{b} \mathcal{R}_{a} \mathcal{R}_{c}=\mathcal{R}_{\text {caba }}=\mathcal{R}_{e}=i d$

Cartan-Maurer equations:

$$
\begin{aligned}
& d \theta^{a}+\theta^{b} \wedge \theta^{c}+\theta^{c} \wedge \theta^{b}=0 \\
& d \theta^{b}+\theta^{a} \wedge \theta^{c}+\theta^{c} \wedge \theta^{a}=0 \\
& d \theta^{c}+\theta^{a} \wedge \theta^{b}+\theta^{b} \wedge \theta^{a}=0
\end{aligned}
$$

The exterior derivative on any three-form of the type $\theta \wedge \theta \wedge \theta$ vanishes, as one can easily check by using the Cartan-Maurer equations and the equalities between exterior products given above. Then, as shown in the previous Section, integration of a total differential vanishes on the "group manifold" of $S_{3}$ corresponding to the $B C_{I}$ bicovariant calculus. This "group manifold" has three independent directions, associated to the cotangent basis $\theta^{a}, \theta^{b}, \theta^{c}$. Note however that the volume element is of order four in the left-invariant one-forms $\theta$.

\section{2 $B C_{I I}$ differential calculus}

Basis of the 2-dimensional vector space of one-forms:

$$
\theta^{a b}, \theta^{b a}
$$

Basis of the 1-dimensional vector space of two-forms:

$$
v o l=\theta^{a b} \wedge \theta^{b a}=-\theta^{b a} \wedge \theta^{a b}
$$

so that:

$$
\theta^{g_{1}} \wedge \theta^{g_{2}}=\epsilon^{g_{1}, g_{2}} \text { vol }
$$

where the $\epsilon$ tensor is the usual 2-dimensional Levi-Civita tensor. Again $f$ vol $=$ vol $f$ since $a b b a=e$. 
Cartan-Maurer equations:

$$
d \theta^{a b}=0, \quad d \theta^{b a}=0
$$

Thus the exterior derivative on any one-form $\theta^{g}$ vanishes and integration of a total differential vanishes on the group manifold of $S_{3}$ corresponding to the $B C_{I I}$ bicovariant calculus. This group manifold has two independent directions, associated to the cotangent basis $\theta^{a b}, \theta^{b a}$.

\subsection{Visualization of the $S_{3}$ group "manifold"}

We can draw a picture of the group manifold of $S_{3}$. It is made out of 6 points, corresponding to the group elements and identified with the functions $x^{e}, x^{a}, x^{b}, x^{c}, x^{a b}, x^{b a}$. $B C_{I}$ - calculus:

From each of the six points $x^{g}$ one can move in three directions, associated to the tangent vectors $t_{a}, t_{b}, t_{c}$, reaching three other points whose "coordinates" are

$$
\mathcal{R}_{a} x^{g}=x^{g a}, \quad \mathcal{R}_{b} x^{g}=x^{g b}, \quad \mathcal{R}_{c} x^{g}=x^{g c}
$$

The 6 points and the "moves" along the 3 directions are illustrated in the Fig. 1. The links are not oriented since the three group elements $a, b, c$ coincide with their inverses.

$$
B C_{I I} \text { - calculus: }
$$

From each of the six points $x^{g}$ one can move in two directions, associated to the tangent vectors $t_{a b}, t_{b a}$, reaching two other points whose "coordinates" are

$$
\mathcal{R}_{a b} x^{g}=x^{g b a}, \quad \mathcal{R}_{b a} x^{g}=x^{g a b}
$$

The 6 points and the "moves" along the 3 directions are illustrated in Fig. 1. The arrow convention on a link labeled (in italic) by a group element $h$ is as follows: one moves in the direction of the arrow via the action of $\mathcal{R}_{h}$ on $x^{g}$. (In this case $h=a b)$. To move in the opposite direction just take the inverse of $h$.

The pictures in Fig. 1 characterize the bicovariant calculi $B C_{I}$ and $B C_{I I}$ on $S_{3}$, and were drawn in Ref. [5] as examples of digraphs, used to characterize different calculi on sets. Here we emphasize their geometrical meaning as finite group "manifolds". 


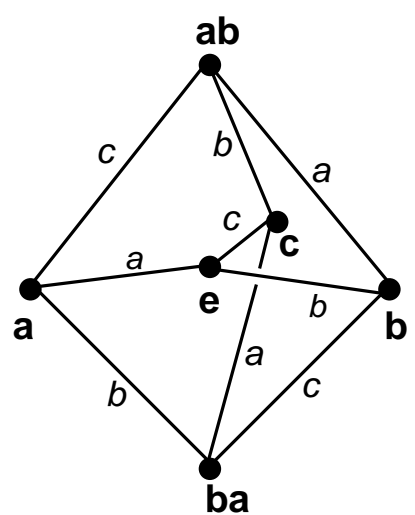

$\mathrm{S}_{3}$ manifold $\left(B C_{1}\right)$
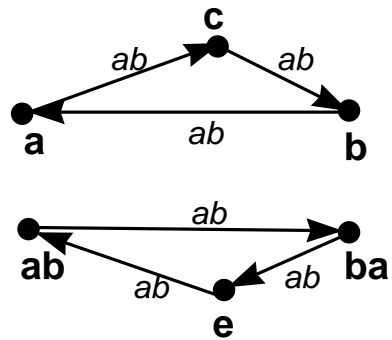

$\mathrm{S}_{3}$ manifold $\left(\mathrm{BC}_{\| I}\right)$

Fig. $1: S_{3}$ group manifold, and moves of the points under the group action

\subsection{Distance matrix}

We define a distance matrix $(\text { dist })_{i, j}$ between two points $i, j$ of the finite group manifold as the minimum number of links that connects them. It is easy to verify that the graphs in Fig. 1 (and more generally any graph corresponding to a bicovariant calculus on a finite $G$ ) are $\operatorname{ad}(G)$ invariant, and therefore dist itself is $\operatorname{ad}(G)$ invariant and can be taken as biinvariant metric. In the case of the connected manifold corresponding to $B C_{I}$ the distance matrix is invertible and given by:

$$
\text { dist }=\left(\begin{array}{llllll}
0 & 1 & 1 & 1 & 2 & 2 \\
1 & 0 & 2 & 2 & 1 & 1 \\
1 & 2 & 0 & 2 & 1 & 1 \\
1 & 2 & 2 & 0 & 1 & 1 \\
2 & 1 & 1 & 1 & 0 & 2 \\
2 & 1 & 1 & 1 & 2 & 0
\end{array}\right)
$$

where rows and columns are ordered as $e, a, b, c, a b, b a$.

For the disconnected $B C_{I I}$ graph we must define also the distance between two disconnected points, and we arbitrarily set it to zero. The resulting distance matrix is also invertible.

\section{Softening $G$ and "gravity" on $S_{3}$}

We have in mind to construct a dynamical theory of vielbein fields whose vacuum solution describes the $G$ manifold. In particular let us take $G=S_{3}$ in the $B C_{I}$ setting. Then the dynamical fields of the theory are collected in the 1 -form vielbein 
$V^{g}$, which is not left-invariant any more since it is a deformation of $\theta^{g}$ :

$$
V^{a}=\sum_{g \in G^{\prime}} V_{g}^{a}(x) \theta^{g}, \quad V^{b}=\sum_{g \in G^{\prime}} V_{g}^{b}(x) \theta^{g}, \quad V^{c}=\sum_{g \in G^{\prime}} V_{g}^{c}(x) \theta^{g}
$$

In addition we consider also the "spin connection" 1 -form $\omega^{g_{1}}{ }_{g_{2}}$ as an independent field (first order formulation). The field equations will determine the expression of $\omega$ in terms of the vielbein field.

We try then to mimic the Einstein-Cartan action of general relativity:

$$
A=\int V_{h_{3}}^{g_{3}} V_{h_{4}}^{g_{4}} \epsilon_{g_{1}, g, g_{3}, g_{4}} \gamma^{g, g_{2}} R_{g_{2}}^{g_{1}} \wedge \theta^{h_{3}} \wedge \theta^{h_{4}}
$$

where $R_{g_{2}}^{g_{1}}$, the "soft" curvature, is given in terms of $\omega$ as in eq. (2.97) and the indices of the $\epsilon$ tensor (defined in (3.7)) are lowered with the (bi-invariant) metric $\gamma_{g_{1}, g_{2}}=\delta_{g_{1}, g_{2}}$. The integrand is a 4 -form (since we chose the $B C_{I}$ calculus on $S_{3}$ ): thus the action is formally identical to the one of general relativity. The one-forms $\theta$ are the vielbeins of $S_{3}$ discussed in the previous Section: they are given one-form fields without dynamics.

\section{Invariances of $\mathrm{A}$}

Consider the field transformations:

$$
\begin{aligned}
& \left(V_{h}^{g^{\prime}}\right)^{\prime}=a_{g}^{g^{\prime}} V_{h}^{g} \\
& \left(\omega^{g^{\prime}}{ }_{h^{\prime}}\right)^{\prime}=a_{g^{g^{\prime}}} \omega^{g}{ }_{h}\left(a^{-1}\right)_{h^{\prime}}^{h}+a^{g^{\prime}} d\left(a^{-1}\right)_{h^{\prime}}^{h}
\end{aligned}
$$

Requiring $A$ to be invariant under these transformations sets some conditions on the $x$ dependent "rotation" matrix $a$. Recalling that the curvature transforms as $\left(R^{g^{\prime}}{ }_{h^{\prime}}\right)^{\prime}=a_{g}^{g^{\prime}} R^{g}{ }_{h}\left(a^{-1}\right)_{h^{\prime}}^{h}$ we find the transformed action $A^{\prime}$ :

$$
A^{\prime}=\int V_{h_{3}}^{k_{3}} V_{h_{4}}^{k_{4}} \epsilon_{g_{1}, g, g_{3}, g_{4}} \gamma^{g, g_{2}} a_{k_{3}}^{g_{3}} a_{k_{4}}^{g_{4}} a_{k_{1}}^{g_{1}} R_{k_{2}}^{k_{1}}\left(a^{-1}\right)_{g_{2}}^{k_{2}} \wedge \theta^{h_{3}} \wedge \theta^{h_{4}}
$$

In the case of usual general relativity, the Lorenz metric and the Levi-Civita tensor are conserved under local Lorenz rotations, and this implies the invariance of the action under local Lorenz transformations.

Here the $\epsilon$ tensor is the one given in (3.9); moreover the $x$ dependent a matrix elements do not commute with the two-form $R_{k_{2}}^{k_{1}}$ as in the usual case.

We will show that if the $a$ matrices entries are taken to be the functions of eq. (2.111) satisfying an additional periodic condition, then the action is invariant under the transformations (4.4).

First, for the adjoint matrices of (2.111) we have $\gamma^{g, g_{2}}\left(a^{-1}\right)_{g_{2}}^{k_{2}}=a_{g_{2}}^{g} \gamma^{g_{2}, k_{2}}$ because of (2.110). Suppose then that the $a$ matrix entries satisfy a "two unequal links" periodic condition:

$$
a^{i}{ }_{j}=\mathcal{R}_{g g^{\prime}} a^{i}{ }_{j}, \quad g \neq g^{\prime}
$$


Then we can bring the $a_{g_{2}}^{g}$ term to the left of the curvature two-form (the $g \neq g^{\prime}$ in (4.6) is due to $\theta^{g} \wedge \theta^{g}=0$ ), and we see that the action $A$ is invariant if:

$$
\epsilon_{g_{1}, g_{2}, g_{3}, g_{4}} a_{k_{1}}^{g_{1}} a_{k_{2}}^{g_{2}} a_{k_{3}}^{g_{3}} a_{k_{4}}^{g_{4}}=\epsilon_{k_{1}, k_{2}, k_{3}, k_{4}}
$$

i.e. if $\epsilon$ is a conserved tensor. But as we have already argued in Section 2.11 this is the case when the $a$ matrix rotates the indices according to the adjoint action of $G$.

Hence we have an action invariant under the local $a d(G)$ transformations, in analogy with the local Lorenz rotations of general relativity.

This action is also invariant under the analogue of general coordinate transformations. Indeed diffeomorphisms along a generic tangent vector $v$ are generated by the Lie derivative $l_{v}=d i_{v}+i_{v} d$. Then under diffeomorphisms the variation of $A$ is given by

$$
\delta A=\int l_{v}(4 \text {-form })=\int\left[d i_{v}(4 \text {-form })+i_{v} d(4 \text {-form })\right]=0
$$

since $d$ (4-form) $=0$ and $\int d$ (3-form) $=0$.

\section{Field equations}

The field equations are obtained by varying the action with respect to the dynamical fields $V_{h}^{g}, \omega^{g}{ }_{h}$. The $\delta \omega^{g}{ }_{h}$ variation yields an equation relating $\omega^{g}{ }_{h}$ to (first derivatives of) the vielbein and its inverse, as in the usual zero-torsion condition of ordinary Einstein-Cartan gravity. Varying with respect to $V_{h}^{g}$ leads to the analogues of Einstein eqs.:

$$
V_{h_{3}}^{g_{3}} \gamma^{g_{0}, g_{2}} R_{g_{2}, h_{1}, h_{2}}^{g_{1}}\left[\epsilon_{g_{1}, g_{0}, g, g_{4}} \epsilon^{h_{1}, h_{2}, h, h_{4}}+\epsilon_{g_{1}, g_{0}, g_{3}, g} \epsilon^{h_{1}, h_{2}, h_{3}, h}\right]=0
$$

where the curvature components $R_{g_{2}, h_{1}, h_{2}}^{g_{1}}$ are defined by $R_{g_{2}}^{g_{1}}=R_{g_{2}, h_{1}, h_{2}}^{g_{1}} \theta^{h_{1}} \wedge$ $\theta^{h_{2}}$.

Note 4.1 : the analogue of a cosmological term

$$
\int \varepsilon_{g_{1}, g_{2}, g_{3}, g_{4}} V_{h_{1}}^{g_{1}} V_{h_{2}}^{g_{2}} V_{h_{3}}^{g_{3}} V_{h_{4}}^{g_{4}} \theta^{h_{1}} \wedge \theta^{h_{2}} \wedge \theta^{h_{3}} \wedge \theta^{h_{4}}
$$

is invariant under the local tangent rotations (4.4) because of property (4.7), and under diffeomorphisms because of (4.8. Adding this term to the action (4.2) allows "vacuum" solutions with $R_{g_{2}, h_{1}, h_{2}}^{g_{1}} \neq 0$.

Note 4.2 : The same action (4.2) can be used in the case of the finite group $Z^{N} \times Z^{N} \times Z^{N} \times Z^{N}$. Here the situation simplifies: for example the $\epsilon$ tensor becomes the usual Levi-Civita tensor, the basic one forms anticommute etc. One then obtains a discretized gravity of the type discussed in ref. [4], with some differences. The field equations are derived from a variational principle, the local symmetry involves functions arbitrary up to a ("two unequal links") periodicity condition, no procedure is used to "localize" the components of the curvature tensor, and no use of the left symmetric tensor product $\otimes_{L}$ is made. 
Note 4.3: the analogue of the topological action:

$$
\int R_{g_{2}}^{g_{1}} \wedge R_{g_{1}}^{g_{2}}
$$

has all the invariances discussed above, even without the two-links periodic condition on the matrices $a(x)$, thanks to $[v o l, f]=0$.

\section{Acknowledgments}

I have benefited from discussions with P. Aschieri and F. Mueller-Hoissen.

\section{A Lie derivative along generic tangent fields}

As discussed in ref. [10] a generic vector field $V$ can be written in terms of the left-invariant tangent vectors $t_{i}$ of eq. (2.75) as $V=a^{i} t_{i}, a^{i} \in F u n(G)$ and defined to act on any $b \in G$ as:

$$
V b=\left(a^{i} t_{i}\right) b \equiv a^{i}\left(t_{i} b\right)
$$

We denote by $\Xi$ the space of vector fields $V$. The product between elements of $\operatorname{Fun}(G)$ and left-invariant tangent vectors $t_{i}$ generalizes to the whole $\Xi:(a V) b \equiv$ $a(V b)$, and

$$
(a+b) V=a V+b V ; \quad(a b) V=a(b V) ; \quad(\lambda a) V=\lambda(a V), \quad \lambda \in \boldsymbol{C}
$$

$\Xi$ is the analogue of the space of derivations on $F u n(G)$. Indeed:

$$
\begin{array}{lr}
V(a+b)=V(a)+V(b), \quad V(\lambda a)=\lambda V(a) & \text { Linearity } \\
V(a b) \equiv\left(c^{i} t_{i}\right)(a b)=t_{i}(a)\left(\mathcal{R}_{i^{-1}} b\right) c^{i}+a V(b) & \text { Leibniz rule }
\end{array}
$$

\section{Inner derivative}

The $i_{V}$ contraction operator is defined by the following properties $\left(V \equiv c^{i} t_{i}\right.$, $a \in \operatorname{Fun}(G), \lambda \in C)$ :

$$
\begin{aligned}
& i_{V}(\rho)=i_{t_{i}}(\rho) c^{i} \\
& i_{V}(a)=0 \\
& i_{V}\left(\theta^{i}\right)=c^{i} \\
& i_{V}\left(\rho \wedge \rho^{\prime}\right)=i_{t_{i}}(\rho) \wedge\left(\mathcal{R}_{i^{-1}} \rho^{\prime}\right) c^{i}+(-1)^{\operatorname{deg}(\rho)} \rho \wedge i_{V}\left(\rho^{\prime}\right) \\
& i_{V}\left(a \rho+\rho^{\prime}\right)=a i_{V}(\rho)+i_{V}\left(\rho^{\prime}\right) \\
& i_{V}(\rho a)=i_{t_{i}}(\rho)\left(\mathcal{R}_{i^{-1}} a\right) c^{i} \\
& i_{\lambda V}=\lambda i_{V}
\end{aligned}
$$




\section{Lie derivative}

Definition:

$$
l_{V} \equiv i_{V} d+d i_{V}
$$

Properties $\left(V \equiv c^{i} t_{i}, a, b, c^{i} \in F u n(G)\right)$ :

$$
\begin{aligned}
& l_{V}(a)=V(a) \\
& l_{V} d \rho=d l_{V} \rho \\
& l_{V}\left(\lambda \rho+\rho^{\prime}\right)=\lambda l_{V}(\rho)+l_{V}\left(\rho^{\prime}\right) \\
& l_{b V}(\rho)=\left(l_{V} \rho\right) b-(-1)^{\operatorname{deg}(\rho)} i_{V}(\rho) \wedge d b \\
& l_{V}\left(\rho \wedge \rho^{\prime}\right)=\rho \wedge l_{V}\left(\rho^{\prime}\right)+l_{t_{i}}(\rho) \wedge\left(\mathcal{R}_{i^{-1}} \rho^{\prime}\right) c^{i}+ \\
& \quad+(-1)^{\operatorname{deg}\left(\rho^{\prime}\right)} i_{t_{i}}(\rho) \wedge\left(\mathcal{R}_{i^{-1}} \rho^{\prime}\right) d c^{i} \\
& l_{t_{i}}\left(\theta^{j}\right)=\boldsymbol{C}_{k, i}^{j} \theta^{k}
\end{aligned}
$$

The proof that the Lie derivative $l_{t_{i}}$ defined as $l_{t_{i}} \rho=\left(i d \otimes t_{i}\right) \Delta_{R}(\rho)$ in (2.116) is equal to $l_{t_{i}}=i_{t_{i}} d+d i_{t_{i}}$ is done by induction on the generic $p$-form $a_{i_{1} \ldots i_{p}} \theta^{i_{1}} \wedge \ldots \wedge \theta^{i_{p}}$, checking first that both definitions give the same result on $\theta^{j}$ (see ref.s $([\mathbb{8}, 10])$ ).

\section{References}

[1] A. Connes, Noncommutative geometry and reality, J.Math.Phys. 36 (1995) 6194; A. Connes and J. Lott, Particle models and noncommutative geometry, Nucl.Phys.Proc.Suppl. 18B (1991) 29.

[2] A. Dimakis and F. Müller-Hoissen, Bidifferential calculi and integrable models, math-ph/9908015.

[3] A. Dimakis, F. Müller-Hoissen and T. Striker, Non-commutative differential calculus and lattice gauge theory, J.Phys. A26 (1993)1927.

[4] A. Dimakis and F. Müller-Hoissen, Discrete Riemannian Geometry, J.Math.Phys.40 (1999) 1518, gr-qc/9808023.

[5] K. Bresser, F. Müller-Hoissen, A. Dimakis and A. Sitarz, Noncommutative geometry of finite groups, J.Phys.A29 (1996) 2705, q-alg/9509004.

[6] F. Bonechi, R. Giachetti, R. Maciocco, E. Sorace and M. Tarlini, Cohomological Properties of Differential Calculi on Hopf Algebras, Proocedings of the Symposium on Quantum Groups of the International Colloquium GROUP21, Goslar 1996, q-alg/9612019.

[7] S.L. Woronowicz,Differential calculus on compact matrix pseudogroups (Quantum groups), Commun. Math. Phys. 122, (1989) 125. 
[8] P. Aschieri and L. Castellani, An introduction to non-commutative differential geometry on quantum groups, Int. Jou. Mod. Phys. A8 (1993) 1667.

[9] L. Castellani, Differential calculus on $I_{S O}(N)$, quantum Poincaré algebra and q-gravity, Comm. Math. Phys. 171 (1995) 383, hep-th 9312179; The lagrangian of q-Poincaré gravity, Phys. Lett. B327 (1994) 22, hep-th 9402033.

[10] P. Aschieri, On the geometry of inhomogeneous quantum groups, Ph.D Thesis, Scuola Normale Superiore di Pisa (1998), math.QA/9805119. 